\title{
Deutsch als Fremdsprache mit digitalen Medien - Versuch einer Zwischenbilanz im Jahr 2008
}

\author{
Dietmar Rösler
}

\begin{abstract}
Zusammenfassung
Diskutiert wird der Stand der Entwicklung des DaF-Lernens mit digitalen Medien unter sechs Gesichtspunkten. Im Bereich der Lehrmaterialentwicklung werden neue Möglichkeiten der Erstellung eines Lehrwerks on demand, in der Lehrwerkanalyse die einer Vereinigung von Werk- und Rezeptionsanalyse aufgezeigt. Danach werden Perspektiven für die Bereiche Einbeziehung der Korpusanalyse, animierte Grammatikdarstellungen und programmiertes Feedback diskutiert. Als sechster Bereich wird das Potenzial der digitalen Medien für kooperatives Lernen besonders unter der Fragestellung behandelt, ob sie einen Beitrag zur Förderung einer weitergehenden Selbstbestimmung der Lernenden leisten können.

Videofassung des Vortrags auf der 36. Jahrestagung des FaDaF in Düsseldorf am 23.05.2008. In: DaF-Netzwerk. Europäisches Netzwerk Deutsch als Fremdsprache (Online). URL: http:// www.daf-netzwerk.org/tagungen/
\end{abstract}

\section{Vorbemerkung}

Diese Zwischenbilanz konzentriert sich auf die folgenden Bereiche: Auswirkungen der digitalen Distributionsweisen auf Fremdsprachenlernen und Lehrmaterialentwicklung, Veränderungen der Lehrwerkanalyse durch die Digitalisierung von Lehrmaterial, Veränderungen, die von Korpusanalyse und animierten Grammatikdarstellungen in das Fremdsprachenlernen hineingetragen werden könnten, Bedeutung von Online-Tutorierung und programmiertem Feedback, Kooperationen von individuellen Lernenden oder Gruppen von Lernenden mit digitalen Medien und Lernertexte für Lerner.
Letzteres, die Diskussion um Lernertexte für Lerner, ist m.E. zur Zeit eine der produktivsten Entwicklungen. Durch die Geschichte des Fremdsprachenunterrichts hindurch hat es immer wieder Ausbrüche aus dem traditionell eher durch Lehrer und Lehrmaterial bestimmten Unterricht mit dem Ziel gegeben, der inhaltlichen Selbstbestimmung ${ }^{1}$ und den Mitteilungsabsichten der Lernenden mehr Raum zu geben bzw. diese seit der sog. kommunikativen Wende zumindest theoretisch $^{2}$ zum Leitgedanken des Fremdsprachenlernens werden $\mathrm{zu}$ lassen. Durch die digitalen Medien scheint m. E. nun die Möglichkeit gegeben zu sein, der

1 Die Attraktivität eines Teils der sog. alternativen Methoden machte ja nicht zuletzt aus, dass diese den Mitteilungsabsichten und inhaltlichen Interessen der Lernenden absolute - und manchmal verabsolutierte - Vorfahrt vor Vermittlungsbemühungen zur Form einräumen, was unter dem Gesichtspunkt der möglichst optimalen Vermittlung der Fremdsprache insgesamt sowohl zu spannenden Neuerungen als auch zu durchaus nicht unproblematischen Nebenwirkungen führen kann (vgl. Rösler 1984: 139-180).

2 Dass zwischen dieser Absicht und dem Alltag der kommunikativen Lehrmaterialentwicklung und des kommunikativen Unterrichts manchmal größere Lücken klaffen, kann an dieser Stelle nicht weiter ausgeführt werden (vgl. dazu Rösler 2008). 
inhaltlichen Selbstbestimmung der Lernenden mehr Raum zur Verfügung zu stellen und damit die berühmte unhintergehbare Künstlichkeit des Fremdsprachenunterrichts doch ein klein wenig zu hintergehen.

\section{Auswirkungen der digitalen Distri- butionsweise: zielgruppengenaueres Lehrwerk on demand?}

Zunächst einmal ist beim Stichwort Distribution die triviale Tatsache festzuhalten, dass ein Text, den man als pdf-Datei verschickt, schneller und kostengünstiger beim Empfänger ankommt als ein dicker Briefumschlag. Entsprechend häufig findet man auf den Internet-Seiten von Verlagen herunterladbare Arbeitsblätter, mp3-Dateien o. ä. zu ihren Lehrwerken ${ }^{1}$. Die darüber hinausgehende, unter lehrmaterialanalytischen Gesichtspunkten interessantere Frage ist die, ob einfach nur der Transport preisgünstiger wird oder ob auch im Hinblick auf die Qualität eines Lehrwerks ein Fortschritt $\mathrm{zu}$ verzeichnen ist. Wenn z. B. von Lehrenden oder Lernenden Audiodateien heruntergeladen werden, die es zwar auch als Kassette oder CD zu kaufen gibt, die aber an bestimmten Orten nicht gekauft wurden, dann ist das unter dem Gesichtspunkt der Betrachtung eines Lehrwerks als >Werk am Produkt selbst hat sich nichts verändert. Aber an den Orten, an denen nun Audiodateien verwendet werden, die bei anderer Distribution vorher nicht zum Einsatz kamen, besteht die Chance, dass sich die Qualität des Unterrichts verändert $^{2}$. Darüber hinaus könnten sich auch die Lehrwerke selbst ändern, wenn durch die digitalisierte Distribution nicht nur das Vorhandene schneller und preisgünstiger an die Lehrenden und Lernenden gebracht wird, sondern wenn über die digitale Distribution hinaus die schnellen Verbindungen dazu genutzt würden, Lehrwerke zielgruppen- und lernergenauer zu gestalten.

So könnten z. B. mehr und bessere Glossare und Lehrerhandbücher entstehen. Man stelle sich ein Lehrerhandbuch nicht als Buch zwischen zwei Deckeln sondern als Datenbank vor, in der sowohl Lehrer, die gerade anfangen zu unterrichten, als auch Lehrer, die mit einem Lehrwerk schon lange arbeiten und deshalb interessante Alternativen zur Routine brauchen, für sie geeignete ausführliche Erklärungen zur Arbeit mit einer Lektion, Erklärungen zum Text, Alternativen zur Arbeit mit diesem Text usw. finden (vgl. zu den verschiedenen möglichen Funktionen von Lehrerhandbüchern und ihrem Beitrag zur Adaption von Lehrmaterial an konkrete Lernergruppen Rösler 1984: 201-221).

Und beim Stichwort Glossare zu einsprachigen, im deutschsprachigen Raum erstellten Lehrwerken kann man sich nicht nur vorstellen, dass es mehr von ihnen gibt zu Ausgangssprachen, für die es bisher noch keine Glossare gab, sondern vor allem, dass diese Glossare anders sind, dass sie nicht lediglich auf eingeschränktem Platz 1:1-Entsprechungen liefern, sondern auch Bildmaterial, Verweise auf Texte, in denen Wortschatz unterschiedlich verwendet wird, Verweise auf korpuslinguistische Recherchen zu einem Wort, Verbindungen zu interkulturellen Erklärungen, die die Grenzen von Wortschatzarbeit und Landeskunde verschwinden lassen usw. usw.

1 Vgl. als ein Beispiel von vielen: http:/ / www.hueber.de/seite/downloads_daf

2 Das wäre also eine erfreuliche Nebenwirkung der andersartigen Distribution, ich weiß nicht, wie frequent dieses Phänomen ist, ich kenne es nur anekdotisch von Aussagen von Kollegen aus vom deutschsprachigen Raum weit entfernten Ländern. 
Erstellt werden könnten derartige Erweiterungen zu bisher eher vernachlässigten Teilen von Lehrwerken dezentral, von regionalen Autoren-Teams, aber auch kooperativ in Ausbildungsseminaren von Lehrern, in Lehrerfortbildungen usw. In derartigen Kontexten sind schon immer Materialadaptionen vorgenommen, Ergänzungen, Hilfestellungen u.ä. produziert worden usw., und danach sind sie in den Schubladen der Beteiligten als Kopien gelandet und vielleicht noch im unmittelbaren Umfeld verwendet worden. Da es nun technisch möglich ist, dezentral zu produzieren und zentral zu dokumentieren, könnte es auch didaktisch möglich sein, diese Vielfalt von Variationen zusammenzubringen zu einer komplexen Dokumentation von Lehrmaterial, die es jedem Nutzer an seinem Ort erlaubt, auf das jeweils zielgruppengenaueste Material zuzugreifen bzw. auf das am wenigsten zielgruppenungenaue und dann entsprechend daran weiterzuarbeiten, diese Überarbeitung evtl. wieder in die Datenbank einzugeben usw.

Wenn man sich Lehrwerke langfristig als aus Datenbanken generiert werdende Entitäten vorstellt ${ }^{1}$, könnte das gerade für das Fach Deutsch als Fremdsprache im Bereich der Lehrmaterialproduktion einen großen Fortschritt darstellen. Denn zu den Defiziten von Deutsch als Fremdsprache im Vergleich z. B. zu Englisch als Fremdsprache gehört es ja, dass Lerner in vielen Teilen der Welt abhängig davon sind, dass im deutschsprachigen Raum qualitativ hochwertige Lehrwerke pro- duziert werden, die dann aber notgedrungen einsprachig ${ }^{2}$ sind und in bestimmten sprach- und kulturkontrastiven Aspekten und bezogen auf das Eingehen auf Lernerfahrungen notwendigerweise nicht zielgruppengenau sein können. Ein derartiges Lehrwerk ginge in seiner bunten Vielfalt weit über das Konzept der Regionalisierung von Lehrwerken aus den frühen 80er Jahren hinaus (vgl. z. B. Bochow/Schroedter-Albers 1981 oder Gerighausen/Seel 1984).

Optimistisch gesehen könnten also die digitalen Kommunikationswege zu einer neuen Art von Produktion von Lehrmaterial führen, zur zielgruppengenaueren Produktion eines Lehrwerks on demand, das aus einer Datenbank generiert wird und das nicht nur bisher vernachlässigte Teile eines Lehrwerks verstärkt, sondern generell die Lehrwerkproduktion qualitativ verändert. Eine mögliche Vorstellung ist, dass man dabei mit einem Kern von didaktisch vielfach variiert dargestellten grammatischen Strukturen arbeitet, um den herum sich eine vielfältige lernerbezogene Peripherie bezogen auf unterschiedliche Themen, unterschiedliche Lernweisen usw. entwickelt (vgl. dazu ausführlicher Rösler 2006a). Pessimistisch müsste man allerdings dagegenhalten, dass die neuen Distributionsmöglichkeiten auch dazu führen könnten, dass sich dominierende weltweit vertriebene einsprachige Lehrwerke noch schneller und intensiver verbreiten, dass es also zu einer Art Lehrwerk-Microsoft kommen könnte.

1 Wobei es bei diesem Gedankengang zunächst einmal nicht darauf ankommt, ob man sich dabei ein lediglich digital vorhandenes Lehrwerk, ein Lehrwerk, dessen Teile ausgedruckt und gebunden an die Lernenden weitergegeben werden, oder Mischungen davon vorstellt. Das Datenbankkonzept zielt zunächst einmal nur darauf ab, zielgruppenangemessenere Vielfalt zu ermöglichen.

2 Abgesehen von Glossaren und einigen Versuchen, zweisprachige Arbeitsbücher hinzuzufügen, die aber die grundlegend einsprachige Ausrichtung dieser Lehrwerke nicht ändern. 
2. Auswirkung der Entwicklung von digitalem Material auf die Lehrwerkanalyse

Die Digitalisierung von Lehrmaterial könnte zu einem Ende der Lehrwerkanalyse, wie man sie heute kennt, führen. Dieser Satz mag etwas dramatisch klingen, ist aber m.E. nicht ganz von der Hand zu weisen. Die Lehrwerkanalyse für $\mathrm{DaF}$ hat unterschiedlich produktive Phasen erlebt, nach Sternstunden mit den Mannheimer Gutachten, den Gutachten Deutsch für ausländische Arbeiter und den entsprechenden hitzigen Diskussionen (vgl. Kommission für Lehrwerke DaF 1978; Engel/Krumm/Wierlacher 1979, die heftigen Reaktionen auf die Mannheimer Gutachten in Heft 2, 1978 der Zeitschrift Zielsprache Deutsch und Barkowski u. a. 1986) gab es längere $>$ Ruhephasen<, in denen die Lehrwerkanalyse zwar ab und zu durch einige Arbeiten vorangetrieben wurde, sich im Alltag aber eher in einem mehr oder weniger entwickelten Rezensionswesen manifestierte. Zur Grundkonstellation der Lehrwerkanalyse für $\mathrm{DaF}$ gehörte schon immer, dass es eine nicht unproblematische personelle Vermischung von Materialmachern und Kritikern gab. Dieses Phänomen ist lange bekannt, wie das folgende Zitat aus dem Jahre 1979 zeigt:

»Es gibt kein Lehrwerk, in dessen Impressum nicht auch die Namen derer zu finden sind, die auch an anderer Stelle an der fachdidaktischen Diskussion teilnehmen. Soll ein Kollege, der an einem Lehrwerk mitgearbeitet hat, das Lehrwerk, an dem ein anderer Kollege beteiligt ist, als fehlerhaft oder ungeeignet abqualifizieren? Das verstößt nicht nur gegen die sguten Sitten im Umgang gebildeter Menschen; man würde auch riskieren, dass der Kollege hingeht und dasselbe tut.« (Neuner 1979: 34)

An dieser Grundproblematik der Lehrwerkanalyse im Bereich $\mathrm{DaF}$ hat sich seither nichts verändert, unter Kontrolle zu halten ist sie nur durch größtmögliche
Transparenz durch Offenlegung von Interessenkollisionen und größtmögliche Zurückhaltung der Materialmacher im Angesicht der Versuchung, bei Artikeln und Vorträgen über ein vermeintlich sneutrales< lehrwerkbezogenes Thema Werbung für ihr aktuelles Werk zu betreiben. Bezogen auf digitales Lehrmaterial ändert sich an dieser Grundproblematik nichts, solange es sich dabei nur um eine $\mathrm{CD}$ zu einem Lehrwerk handelt oder um eine frei zugängliche oder kostenpflichtige Seite mit Ergänzungen im Netz. Hinzu tritt im digitalen Kontext m. E. nun allerdings eine Veränderung, die die Lehrwerkanalyse, wie sie bisher betrieben wird, beendet: entweder findet sie in Zukunft nicht mehr statt oder sie erreicht eine neue Qualität.

Der Lehrwerkanalytiker aus den Zeiten von Deutsch aktiv ging in die Bibliothek oder die Buchhandlung, besorgte sich Bücher und Kassetten, später auch CDs, und analysierte los. Er betrieb zumeist Werkanalyse und keine empirisch fundierte Rezeptionsanalyse, was sicher zu bedauern war, aber er hatte immerhin seinen Analysegegenstand - das Werk vollständig vor sich. Was ist aber das Äquivalent zum >Werk < bei einem digitalen Lehrwerk? Wenn das digitale Lehrwerk ein ernst zu nehmendes Komplettangebot sein will, dann muss es beim jetzigen Stand der Entwicklung von programmiertem Material mit einer Tutorierungskomponente versehen sein, und die Qualität dieser Tutorierung sagt wahrscheinlich mehr über die Qualität des Gesamtangebots aus als die Qualität einzelner Texte und Aufgaben. Wer also einen Online-Kurs als Lehrwerk analysieren will, kann sich nicht einfach nur das Material anschauen, er muss sehen, wie dieser Kurs tatsächlich abläuft.

Optimistisch betrachtet und positiv gewendet würde dies bedeuten, dass es in der Lehrwerkanalyse endlich zu empi- 
rischer Rezeptionsforschung kommen muss, die das Lehrwerk in Interaktion mit den Lernenden und Lehrenden betrachtet. Konsequent zu Ende gedacht hätte man es dabei sogar mit dem Ende der Lehrwerkanalyse durch die Aufhebung auf eine höhere Ebene zu tun. Da es sich bei der Analyse eines tutorierten Online-Werks in Aktion eigentlich um die Analyse dessen handelt, was als Äquivalent zum traditionellen Unterricht im Klassenzimmer $\mathrm{zu}$ betrachten ist, würde die Lehrmaterialanalyse aufgehen in empirische Unterrichtsforschung, die ja eigentlich die Interaktion von Lehrenden, Lernenden und Material zum Gegenstand hat, auch wenn die Rolle des Materials dabei nicht immer die ihr eigentlich gebührende Beachtung findet. So ein >Ende<, ein Ausbrechen aus dem selbstgewählten Getto des werkanalytischen Vorgehens, wäre eine erfreuliche Weiterentwicklung der Lehrwerkanalyse, bei der die werkanalytischen Vorgehensweisen nicht verschwinden, sondern als notwendige Blicke auf Struktur, Themen usw. von Material in das rezeptionsanalytische Vorgehen integriert würden. Aktuelle Versuche, Lehrmaterial in Aktion zu analysieren, beziehen sich auf die Analyse des Umgangs von Lernenden mit Selbstlernmaterial und zeigen, wie komplex und methodisch raffiniert man sich diese Wende der Lehrwerkanalyse vorzustellen hätte (vgl. Schmidt 2007 oder Würffel 2006).

Pessimistisch, aber m. E. leider wohl nicht ganz unrealistisch betrachtet könnte das verstärkte Aufkommen von digitalen Kursen hingegen dazu führen, dass es überhaupt keine ernsthafte Lehrwerkanalyse mehr geben wird. Mein subjektiver Eindruck von Fachtagungen der letzten zehn Jahre ist, dass die entsprechenden Sektionen von mehr oder weniger offen als solchen deklarierten Präsentationen von Materialien, meist unter einer an die Fragestellung des Rahmenthemas angelehnten Thematik, dominiert wurden und kritische lehrmaterialanalytische Auseinandersetzungen in der Minderheit blieben. Diese Diskurshoheit der >Bastler ist vielleicht ein - notwendiges ${ }^{1}$ - Übergangsphänomen, es wird aber verschärft durch die Frage nach dem Zugang. Zugang erhält man bei OnlineKursen zu ausgewählten Probestücken, diese dienen der Werbung, nützen aber nichts für eine distanzierte Analyse. Wenn Lehrwerkanalyse im digitalen Zeitalter überleben soll, muss also nicht nur dafür gesorgt werden, dass die Präsentation von Online-Materialien durch die Produzenten auch klar als solche erkenntlich ist, sondern auch dafür, dass Zugänge zu den Kursen für Forscher, die nicht identisch mit den Machern sind, selbstverständlich sind, so dass eine Analyse der tatsächlichen Nutzungen von Online-Kursen möglich wird.

\section{Interessante aktuelle Tendenzen: ani- mierte Grammatik und Einbeziehung der Korpusanalyse}

Aus Platzgründen nur ganz kurz sollen in diesem Kapitel exemplarisch zwei ganz unterschiedliche Aspekte angesprochen werden, die zeigen, wie der Fremd-

1 Um keine Missverständnisse aufkommen zu lassen: Es ist sehr wichtig und bei so komplexen Gegenständen wie digitalen Lehrmaterialien unbedingt notwendig, dass sich Macher austauschen, gute Ideen verallgemeinern und so weitgehend wie möglich kooperieren. Dazu bedarf es des Diskurses der Macher. Diese Diskussionen sind aber systematisch zu trennen von empirischen lehrwerkanalytischen Forschungen zu digitalem Lehrmaterial, so wie auch die Präsentation von traditionellem Print-Material auf Kongressen zu trennen ist und oft auch getrennt wird von lehrwerkanalytischen Vorträgen. 
sprachenvermittlung durch Digitalisierung neue Wege erschlossen werden können, die Frage nach der Relevanz animierter Grammatik und die nach der Reichweite der Einbeziehung der Korpusanalyse.

\subsection{Animierte Grammatik}

Wenn man auf die Entwicklung von Lehrwerken zurückblickt, lässt sich feststellen, dass im Laufe der Zeit verstärkt versucht worden ist, mit Visualisierungen $\mathrm{zu}$ arbeiten, die den Lernenden das Verstehen erleichtern sollen (vgl. die Beispiele in Funk/König 1991). Wenn man sich hingegen die ersten Grammatikdarstellungen im Netz anschaut, blickt man meist auf Bleiwüsten, auf kopierte komprimierte Grammatikdarstellungen, die hinter die Qualität der Visualisierungen in >Papierwerken zurückfielen. Deren Standard müssten die digitalen Darstellungen also auf jeden Fall zunächst nachvollziehen. Aber durch die Digitalisierung können die Bilder das Laufen lernen, d.h. prinzipiell ist zu überlegen, inwieweit eine animierte Grammatik eine Erleichterung und Lernhilfe für die Lernenden darstellen könnte. Ein beliebter grammatischer Gegenstand für eine Animierung sind z.B. Wechselpräpositionen ${ }^{1}$, eine Figur stellt etwas auf einen Tisch, steht auf einem solchen o. ä. Für einen Selbstlerner, der allein und ohne Interaktion im Klassenzimmer Deutsch lernt, ist so ein kleiner Film, der das Prinzip der Wechselpräpositionen verdeutlicht, eventuell hilfreich. Aber in jedem Klassenraum kann ein Lehrer die Wechselpräpositionen ohne Schwierigkeiten demonstrieren, es gibt genug Leute und Gegenstände, die in einen Raum gehen, in einem Raum sind, etwas in eine Tasche, unter einen Tisch oder wohin auch immer stellen oder legen, worauf die Gegenstände dann unter dem Tisch sind usw. Wenn man weiß, wie aufwändig es ist, gute animierte Grammatikdarstellungen zu produzieren, dann stellt sich zunächst die Frage, für welche grammatischen Phänomene sich der Aufwand eigentlich lohnt.

M. E. sollten Visualisierungen zunächst besonders dazu benutzt werden, kognitiv explizit kaum noch zu vermittelnde Konzepte leichter zugänglich $\mathrm{zu}$ machen. So könnte ein schwieriges Kapitel der deutschen Grammatik, die Stellung im Mittelfeld ${ }^{2}$, von einer animierten Grammatik profitieren, die Komplexität ganzheitlicher darstellen kann als explizite Erläuterungen zu Neuem und Bekanntem, zu Links- und Rechtstendenz, Pronominalisierungen usw. Man stelle sich z. B. eine Straße vor, auf der unterschiedliche Arten von Parkverboten und Halteeinladungen vorhanden sind und unterschiedliche Angaben- und Ergänzungsautos vorbeigefahren kommen, die je nach pronominalem oder nominalem Zustand an bestimmten Stellen parken, von einem Parkwächter von bestimmten Stellen verwiesen werden usw. usw. Wenn Animierungen dazu führen, dass etwas Komplexes und Schwieriges leichter verstanden wird, dann tragen sie zu einer neuen Qualität des Lernens bei. Wenn Selbstverständliches oder leicht Verständliches illustriert wird oder wenn die Aufmerksamkeit der Lernenden durch eine Animierung, die eher Ornament ist, vom eigentlich $\mathrm{zu}$ Lernenden abgelenkt wird, dann

1 Vgl. z. B. http:/ / www.passwort-deutsch.de/lernen/band2/lektion8/aktivitaet02. htm\#

2 Bei diesem Gegenstand scheint mir inzidentelles Lernen einem kognitiv expliziten Zugang vorzuziehen zu sein, der entweder zu stark simplifiziert (Beispiel: tekamolo) oder der leicht das Ausmaß einer Linguistik-Vorlesung erreicht. 
wird eher ein Problem als eine Hilfestellung produziert. Animationen eignen sich zur Darstellung sequenzieller oder kausaler Sachverhalte, aber nur solange auch dies nicht $\mathrm{zu}$ einer Reizüberflutung oder Ablenkung führt.

»An der falschen Stelle eingesetzt nehmen sie daher wichtige Ressourcen von anderen essenziellen Verarbeitungsaufgaben weg. ... Die scheinbar leichte Verständlichkeit, die durch multimediale Animationen häufig suggeriert wird, verhindert ... richtiges Verstehen.«(Roche/Scheller 2004: 14)

\subsection{Korpuslinguistik für den und im DaF-Unterricht?}

Ein zweiter Bereich, der hier nur kurz erwähnt werden soll, ist das Potenzial, das durch die Verbindung von Computertechnologie und linguistischer Methodik für den Fremdsprachenunterricht nutzbar gemacht werden kann bzw. könnte, das Potenzial der Korpuslinguistik. Diese ist in der Anglistik weiter entwickelt als in der Germanistik, das gilt sowohl für ihren Kernbereich, die Linguistik, als auch für Versuche, sie für das Fremdsprachenlernen produktiv zu machen (vgl. z. B. Mukherjee 2004 und Mukherjee/Rohrbach 2006). Aber auch im Bereich DaF wird durch die 2008 in der Zeitschrift Deutsch als Fremdsprache eröffnete Reihe von Beiträgen (vgl. Fandrych/Tschirner 2008 und Meißner 2008) die Diskussion systematisiert, und mit FALKO wächst an der Humboldt Universität ein annotiertes Fehlerkorpus für DaF heran (vgl. Lüdeling u. a. 2008).

In der Linguistik hat die Korpusanalyse eine methodische Neuorientierung bewirkt, an die Stelle einzelner Belegstellen oder der Introspektion des Muttersprachlers tritt die Analyse immer größerer Mengen von Daten. Für den Fremdsprachenunterricht ist die Korpuslinguistik auf zwei Ebenen interessant.
Zunächst ist sie, wiederum auf zwei Ebenen, Lieferantin von Informationen. Zum einen stellt sie empirisch überprüfte Aussagen zur Frequenz von sprachlichen Phänomenen zur Verfügung, von denen Lehrmaterialmacher zumindest jenseits von B1 bei der Erstellung von spezielleren Materialien profitieren können. Zum anderen kann die Korpusanalyse da, wo sie Lernerkorpora analysiert, hilfreiche Daten liefern; annotierte Fehlerkorpora können über die große Zahl zu einer interessanten Ergänzung psycholinguistischer Erwerbsforschung werden. In diesen beiden Fällen ist die Korpuslinguistik eine interessante Bezugswissenschaft für die Fremdsprachendidaktik.

Offener ist $\mathrm{m}$. E. zur Zeit noch die didaktisch weitergehende Frage, ob und inwieweit ein korpuslinguistisches Vorgehen auch Teil des institutionell gesteuerten Lernens werden sollte, z. B. dadurch, dass Lerner größere Mengen von sprachlichem Material unter für sie zum jeweiligen Stand ihres Spracherwerbs relevanten Gesichtspunkten durchforsten, inwieweit also die Korpuslinguistik einen Beitrag zum entdeckenden Lernen leisten kann. Dies scheint zunächst eine Frage zu sein, die für Lernende in der Mittelstufe und Oberstufe von Interesse ist, z. B. bei der Beschäftigung mit Kollokationen (vgl. z. B. Ludewig 2005). Wie produktiv ein korpuslinguistisches Vorgehen für DaF-Lernende tatsächlich ist, wird sich erst sagen lassen, wenn entsprechende Unterrichtsforschung vorliegt. Perspektivisch festhalten kann man aber, dass ein korpuslinguistisches Vorgehen zumindest theoretisch das Potenzial in sich trägt, selbstbestimmtes Lernen, das in der Fremdsprachendidaktik zumeist stärker auf Inhalte bezogen diskutiert wird, auch im Bereich der eher formbezogenen Fragestellungen voranzutreiben. 


\section{Tutorierung und programmiertes Feedback}

Feedback ist kein neues Thema der Fremdsprachendidaktik, es gewinnt aber im Kontext der stärkeren Individualisierung, die durch digitale Medien möglich wird (vgl. dazu genauer Rösler 2006b) ${ }^{1}$, besondere Bedeutung. Feedback geben traditionell Lehrer und Mitlernende. Wenn das Lernen nicht mehr in einem Raum stattfindet, in dem Lehrende und Lernende gleichzeitig anwesend sind, kann der Lehrer durch einen Online-Tutor ersetzt werden, was zunächst einmal den Verlust einiger nonverbaler Feedback-Möglichkeiten mit sich bringt, im Hinblick auf Erreichbarkeit für individuell gewählte Lernzeitpunkte aber evtl. für die Lernenden auch eine Verbesserung darstellen kann. ( $\mathrm{Zu}$ den verschiedenen Besetzungen des Begriffs Tutor im Kontext der Diskussion um das Fremdsprachenlernen mit digitalen Medien vgl. Rösler 2004: $194 \mathrm{ff}$.). Wie gut oder wie schlecht ein Online-Tutor ist, hängt nicht zuletzt davon ab, ob er ein Sprachlernhelfer und Sprachberater ist oder eine schlecht bezahlte Hilfskraft, die nur >Häkchen macht<. Wenn ein Team gut ausgebildeter Online-Tutoren Selbstlernenden $\mathrm{zu}$ verschiedenen Zeitpunkten zur Verfügung steht und sie angemessen berät, dann ist Online-Tutorierung zumindest eine ernst zu nehmende Angelegenheit.

Ein Phänomen, über das noch relativ wenig bekannt ist, das aber m.E. im Kontext der digitalen Medien eine größere Rolle spielen wird als bisher, ist das Feedback durch Mitlerner, besonders überall da, wo Lernende individuell an einem Lernprogramm arbeiten, sich aber mit anderen Lernenden im gleichen
Raum befinden. Hier kann das schon immer vorhandene Tuscheln mit dem Nachbarn im Klassenzimmer, wenn man als Lerner mal wieder nicht verstanden hat, was der Lehrer gesagt hat, ihn aber nicht unterbrechen wollte, oder wenn man sich schnell vergewissern möchte, ob man eine Aufgabe richtig gelöst hat, $\mathrm{zu}$ einem immer wichtiger werdenden Bestandteil von Lernprozessen werden. In einem digitalen Kontext, in dem das Lernprogramm nur eines der offenen Fenster auf dem Computer ist und die Lernenden sich gegenseitig bei zeitgleich vorhandenen Online-Wörterbüchern, Grammatiken, beliebigen Informationsseiten usw. informieren und beraten können, könnte mit derartigen Peer-Beratungen ein qualitativ sehr interessanter Schritt liegen in Richtung eines vielleicht in Zukunft wachsenden Bereichs des, paradox formuliert, kooperativen Alleinlernens (vgl. dazu die Untersuchung von Schmidt 2007 zum gemeinsamen Arbeiten mit Selbstlernsoftware).

Beim Thema Feedback und digitale Medien stellt sich neben der Erforschung von durch Computer vermittelten Rückmeldungen von Menschen an andere Menschen vor allem die Frage, was für Alleinlernende eigentlich ein sinnvolles Feedback des impliziten, also des in das Material programmierten, Tutors ist.

Programmiertes Feedback basiert auf dem Abgleichen von Mustern. Je mehr ein Materialmacher vorhersieht, welche Fehler Lernende machen werden, und je mehr Energie er darauf verwendet, auf diese Fehler zu reagieren, desto differenzierter kann sein Feedback aussehen. Das gilt für simple Hot Potatoes-Übungen (vgl. http:/ /hotpot.uvic.ca/) ebenso wie für elaboriertere programmierte Aktivitäten.

1 M. E. wird durch die digitalen Medien sowohl eine stärkere Individualisierung als auch eine Stärkung von Kooperationen beim Fremdsprachenlernen möglich. 
Es handelt sich dabei um eine Fleißarbeit, die für die Macher frustrierend sein kann. Wer viel Energie in eine schöne FlashPräsentation steckt, kann sicher sein, dass die Lernenden die Präsentation sehen und hoffentlich auch gut finden. Wer sehr viel Zeit in die Antizipation von Lernerreaktionen und entsprechende Rückmeldungen der Lernsoftware steckt, weiß nicht, ob irgendetwas von dieser vielen Arbeit überhaupt wahrgenommen wird. Das führt dazu, dass bei vielen Übungen das Feedback sicher weniger differenziert ist, als es technisch sein könnte. Bei offenen Aufgaben ${ }^{1}$ bleibt ohnehin, wenn kein Online-Tutor zur Verfügung steht, an den Lösungen eingesandt werden können, nur die Möglichkeit der Musterantwort, und Musterantworten sind eine zweischneidige Sache. Eine ihrer unerwünschten Nebenwirkungen kann sein, dass ein Lerner, der eine interessante Lösung produziert hat, sehr verunsichert wird, weil er seine gefundene Lösung nicht in Beziehung zur Musterlösung setzen kann.

Der hohe Arbeitsaufwand für das Vorhersehen von und Reagieren auf unterschiedliche Lernereingaben verbunden mit der mangelnden Sichtbarkeit des Feedback mag einer der Gründe dafür sein, dass das Feedback bei manchen Aufgaben in der Lernsoftware stark verbesserungswürdig zu sein scheint. Darüber hinaus ist aber auch nicht hinreichend geklärt, wann für welche Lernenden bei welcher Aufgabe welche Art von Feedback denn eigentlich gutes Feedback ist.

$\mathrm{Zu}$ Anfangszeiten der Lernsoftware-Entwicklung las man manchmal, Feedback solle freundlich sein und nicht verschre- cken. Aber das kann zu Problemen führen, wie mein Selbstversuch aus dem Jahre 1998 zeigte:

»Bei einer graphisch sehr ansprechend gestalteten Übung, bei der ich Substantiven einen definiten Artikel zuordnen muß, macht es offensichtlich einen Unterschied, welchen falschen Artikel ich verwende, denn manchmal erhalte ich bei Fehlern ein aufmunterndes >stimmt nicht ganz<, manchmal ein strenges >das stimmt nicht<. Sollte es hier eine von der germanistischen Linguistik noch nicht entdeckte Tendenz zum semiakzeptablen Zweitgenus für Substantive geben, deren Angabe die freundlichere Fehlermeldung auslöst?« (Rösler 1998: 11)

In einer derartigen Situation muss ein Lerner ins Grübeln kommen, er muss annehmen, dass die unterschiedlichen Reaktionen des Programms bedeuten, dass er unterschiedlich schlecht reagiert hat. Die programmierte Freundlichkeit kann also dazu führen, dass im Kopf der Lernenden fehlgeleitete Verallgemeinerungen in Gang kommen.

Ein anderes Kriterium für die FeedbackGestaltung, das häufig anzutreffen ist, könnte formuliert werden als: je ausführlicher desto besser. Wenn das Feedback des Programms, wie es in den Anfangszeiten von Übungen auf dem Computer manchmal vorkam, einfach lautete, "Einige der Antworten sind nicht ganz richtig«, dann wäre sicher ein ausführlicheres Feedback besser gewesen. Aber was heißt ausführlich? Soll eine Grammatikübung im Feedback z. B. eine Erklärung liefern, einen Link auf eine Seite, in der das Phänomen dargestellt wird? Oder ist das nicht viel zu aufwändig für einen Lerner, der bei einer geschlossenen Übung nur schnell wissen möchte, ob was er angeklickt hatte richtig oder falsch

1 Die Auswahlmöglichkeiten, die Autorensysteme anbieten, führen wohl auch dazu, dass im Netz mehr geschlossene Aufgaben zur Verfügung stehen, als man das für didaktisch sinnvoll halten mag. 
war. In der Untersuchung von Schmidt 2007 drückte sich Feedbackverdrossenheit dadurch aus,

»dass viele Schülerinnen als Folge der beschriebenen Negativerfahrungen die erscheinenden Meldungen häufig entweder vollständig ignorieren, indem sie sie ungelesen sofort nach ihrem Erscheinen wegklicken, oder aber nur sehr oberflächlich wahrnehmen. So kommt es natürlich häufig vor, dass durchaus gute, informierende, fehlerspezifische Kommentare ebenfalls vollständig unbeachtet bleiben« (Schmidt 2007: 291).

Wichtig ist in der momentanen Situation wohl, dass die Antwort auf die Frage, was ein angemessenes Feedback ist, lernerabhängig gestellt werden muss: Die sogenannte Generation Netzkind, für die das Etablieren sozialer Beziehungen im Netz ebenso zum Alltag gehört wie die schnelle Suche nach Informationen im Netz und das schnelle Wegklicken von etwas, was nicht sofort als relevant angesehen wird, wird vielleicht sehr anders mit kognitiv arbeitendem Feedback umgehen als ein älterer Lernender mit anderen Computer- und Lernerfahrungen.

Befänden wir uns in einer Naturwissenschaft und nicht in einem Niemandsland der Forschungsförderung wie der Fremdsprachendidaktik, dann hätten wir sicher schon längst ein millionenschweres Grundlagenforschungsprogramm aufgebaut, bei dem versucht würde zu ermitteln, welche Kriterien für programmiertes Feedback belastbar sind, bezogen auf unterschiedliche Lernertypen, Lernziele, Übungstypen usw. Die häufig beklagte fehlende Breite der empirischen Fremdsprachenforschung ist auch hier ein Hindernis dafür, dass über Argumentationen entlang der reflektierten Erfahrung kaum hinausgegangen wird (vgl. aber z.B. Arbeiten wie Nagata 1993 zu Ausführlichkeit von Feedback und Lernerfolg oder Pujola $2001 \mathrm{zu}$ unterschiedlichen Verwendungsmöglichkeiten und Zweischrittigkeit). Was man jedoch einigermaßen sicher sagen kann, ist, dass ganz langfristig im Bereich Feedback für programmiertes Material ein großer qualitativer Schritt voran erst gemacht werden kann, wenn Pattern-Matching und die Antizipation von Lernereingaben ergänzt und evtl. einmal sogar ersetzt werden durch eine intelligente Analyse der Lernereingaben, d. h. wenn Fremdsprachendidaktik und Forschung zur künstlichen Intelligenz auf einer Ebene zusammenkommen, die über die Analyse von Einzelbeispielen hinausgeht. Das wird aber noch eine sehr lange Zeit auf sich warten lassen (vgl. dazu Gamper/Knapp 2002 oder Puskas 2005).

\section{Kooperatives Arbeiten mit digitalen Medien}

Kooperationen gab und gibt es vor und außerhalb des Arbeitens mit digitalem Material. Tandems gab es, bevor es ETandems gab, Klassenkorrespondenzen, bevor per E-Mail usw. die Raumüberwindung zwischen Lernergruppen an verschiedenen Orten beschleunigt wurde $^{1}$ (vgl. als Überblick Rösler 2004: 49-69). Rasant geändert haben sich in den letzten 10 Jahren die technischen Möglichkeiten für Kooperationen. Mussten vor ca. 10 Jahren für die Untersuchung von Tamme 2001 zur Ausbildung von Online-Tutoren noch bei vielen der Teilnehmer Modems installiert

$1 \mathrm{Vgl}$. als Überblick über laufende Projekte die Sammlung im Netz http:// www.schule.de/englisch/DaF.htm von Reinhard Donath, der für Einstieger auch sehr nützliche Hinweise für das Gelingen von Kooperationsprojekten bereitstellt: http:/ / www.englisch.schule.de/tipps_neu.htm\#zehn. 
werden, sind heute alle Beteiligten am Gießener elektronischen Praktikum (vgl. Würffel 2004) in der Lernplattform Moodle zu Hause. Das bedeutet zunächst einmal nur, dass technisch über den einen Modus der schriftlichen Interaktion per E-Mail hinaus inzwischen eine ganze Palette von Kommunikationsmöglichkeiten bereit steht, synchron und asynchron, geschrieben und gesprochen, in Wikis, Chats, Foren oder per Skype. Die didaktisch relevante Frage lautet, ob dabei der didaktische Fortschritt mit dem technologischen Fortschritt Schritt gehalten hat.

Mit E-Mail und Chat waren digitale Kommunikationsweisen in den Fremdsprachenunterricht eingetreten, die in Bezug auf konzeptionelle Schriftlichkeit und Mündlichkeit nicht immer einfach einzuordnen sind und die für die Fremdsprachendidaktik unter dem Gesichtspunkt der Diskussion der Förderung von Mündlichkeit und Schriftlichkeit interessant waren (vgl. die Analyse von Lemnitzer/ Naumann 2001; zu den Besonderheiten der Kommunikation in didaktischen Chats vgl. Platten 2003). Problematisch war es m. E. zu glauben, dass mit den E-Mails und Chats eine Renaissance des Schreibens stattgefunden hatte, stattgefunden hatte aber auf jeden Fall eine Renaissance des Tippens. Hier kann nun durch die Erweiterung der technischen Möglichkeiten eine Ausdifferenzierung erfolgen. Mit Skype könnte es eher als mit den getippten Chats tatsächlich dazu kommen, dass die Förderung der mündlichen Kommunikationsfähigkeit im Fremdsprachenunterricht stärker in den Fokus des Lernens mit digitalen Medien tritt, und mit Wikis (zum Schreiben von DaF-Lernenden im Wiki vgl. Platten 2008) und Blogs könnte es nun tatsächlich zu einer Renaissance des Schreibens und dabei durch das Potenzial der social software sogar zu einer Stärkung des kooperativen Schreibens kommen (vgl. dazu ausführlicher Würffel 2008).

Digitale Kooperationsprojekte haben zumeist versucht, zumindest die inhaltliche Selbstbestimmung der Lernenden möglichst weitgehend unbeschädigt $\mathrm{zu}$ lassen, der Selbstbestimmung der Arbeitsweisen waren durch die 'Sachzwänge< der vorhandenen Kommunikationsweisen, zunächst beschränkt auf EMail, Grenzen gesetzt. Durch die Vielfalt der technischen Optionen in Lernplattformen ist in Kooperationsprojekten jetzt eine weitergehende Selbstbestimmung von Arbeitsweisen der Wahl, eines synchronen oder asynchronen Vorgehens usw. durch die Lernenden möglich; einer Zwischenbilanz in zehn Jahren bleibt es vorbehalten festzuhalten, ob diese größere Freiheit der Wahl der Mittel zu größerer Selbstbestimmung bei der Wahl der Arbeitsweisen geführt hat.

Auch bei größerer Begeisterung für die sich bietenden Möglichkeiten von Lernplattformen sollte man jedoch nicht vergessen, dass die Grundprobleme sprachund kulturgrenzenüberschreitender Kommunikation durch diese nicht aus der Welt geschafft worden sind, so dass auf die Gefahren in jedem einzelnen Projekt ebenso wie in der Aus- und Fortbildung von Fremdsprachenlehrern geachtet werden muss (vgl. dazu z. B. MüllerHartmann 2000 und O'Dowd/Ritter 2006). Trotz der erweiterten Kommunikationsmöglichkeiten gilt weiterhin der Gemeinplatz: Wenn man sich nichts zu sagen hat, ist es egal, wie luxuriös die Lernplattform ist, in der man sich nichts zu sagen hat. Die entscheidende Frage lautet weiterhin: Haben Lernende in Kooperationsprojekten anderen Lernenden etwas mitzuteilen? 


\section{Lernertexte für Lerner}

Dass Lernende anderen Lernenden oder ihrer Umwelt etwas mitteilen wollen und dass dieses Mitteilungsbedürfnis die Basis des Fremdsprachenlernens sein sollte, ist kein durch digitale Medien aufgekommenes Konzept, sondern Grundannahme aller Versuche, emanzipatorischen Fremdsprachenunterricht $\mathrm{zu}$ machen, und Ausgangsüberlegung der Projektdidaktik. Inwieweit tragen durch digitale Medien realisierte Interaktionen dazu bei, diese Grundannahme stärker im Unterrichtsalltag zu verankern? Diese Frage soll in zwei Teilfragen überführt werden, in die Frage, inwieweit >lehrmaterialfreie selbstbestimmte Textproduktion durch social software in anderer Weise möglich ist als bisher, und in die nicht so offensichtliche, m.E. aber für die Entwicklung des gesteuerten DaF-Lernens hochrelevante Frage danach, ob durch die Digitalisierung auch im traditionellen Lehrmaterialkonzept so etwas wie eine stärkere Einbeziehung von Lernertexten möglich ist.

\subsection{Freie Lernertexte im Netz}

Abb. 1 zeigt einen von einem Lerner ins Internet gestellten Text.

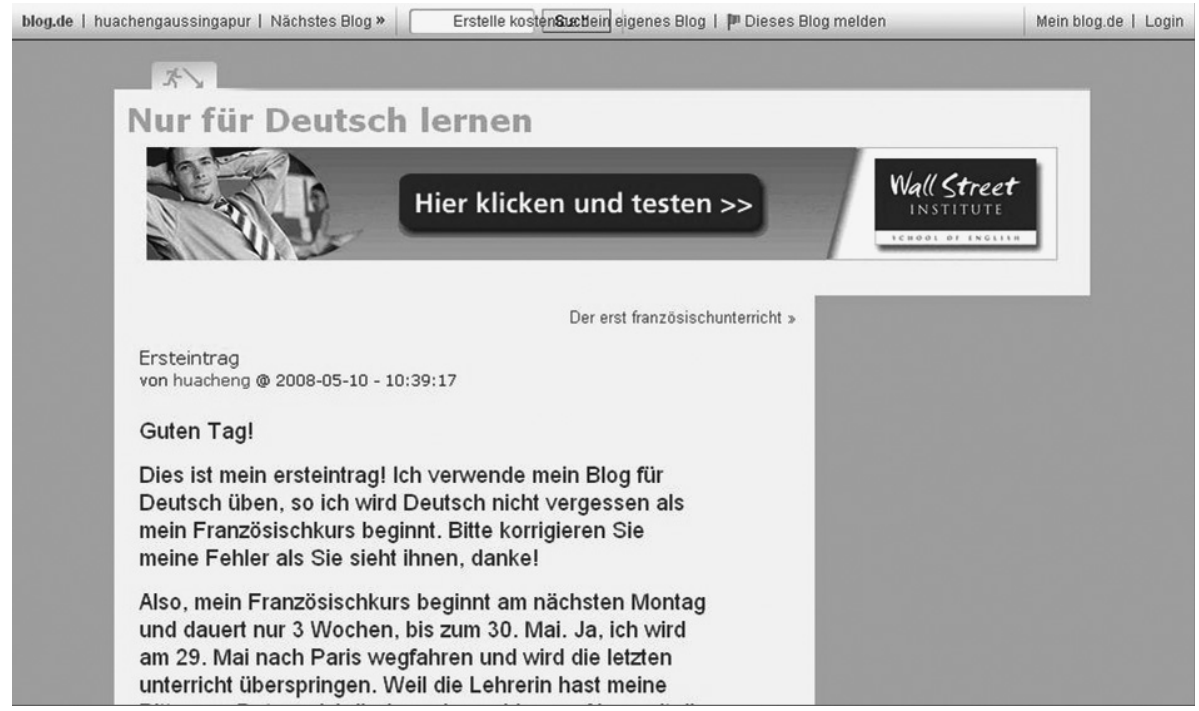

Abb. 1: Von einem DaF-Lerner außerhalb des institutionellen Lernens ins Netz gestellter Text. Quelle: http://huachengaussingapur.blog.de/2008/05/10/ersteintrag-4155058

(Abrufdatum: 21.5.2008)

Wenn derartige Texte von Lernenden produziert und der Welt kund getan werden, ohne dass dieses eine Beziehung zum Unterricht hat, dann handelt es sich zunächst einmal um nichts anderes als um eine mediale Erweiterung von Transfermöglichkeiten. So wie ein Lerner früher einen muttersprachlichen
Touristen im Urlaub angesprochen haben mag, um sein erworbenes Deutsch auszuprobieren, und dabei keinesfalls korrekt gesprochen hat, so kann er jetzt die ganze Welt ansprechen, um sein erworbenes Deutsch auszuprobieren, und er hofft natürlich, dass die Welt ihn zur Kenntnis nimmt. 
Etwas anderes ist es, wenn diese Arten von Aktivitäten innerhalb des Klassenzimmers in die Wege geleitet werden, wenn also Lehrende die Lernenden dazu bringen, sich der Welt mitzuteilen. Hier kann m. E. die Idee des freien Sich-Mitteilens in Konflikt geraten mit der Schutzfunktion, die Lehrende einer Gruppe von Lernenden gegenüber haben, denn im Gegensatz zur Ermunterung zu selbstbestimmtem Schreiben, das zu Plakaten im Klassenzimmer, einer Lesung auf einem Elternabend oder zu einem Brief an eine Partnerklasse führte, liest plötzlich die ganze Welt mit, wenn die Texte nicht entsprechend per Intranet oder sonstige Schutzmechanismen mit einer Zugangsbeschränkung versehen sind. Nun könnte man einwenden, dieser Verweis auf die Schutzfunktion sei sehr altmodisch, wir lebten schließlich in einer Welt des medialen Exhibitionismus, und von daher sind diese Bedenken wahrscheinlich tatsächlich ein bisschen altväterlich, trotzdem sollte man nicht ganz außer Acht lassen, dass ein gegen sprachliche Normen verstoßender oder inhaltlich selbstentblößender Text natürlich auch gegen den Verfasser verwendet werden kann, z. B. eine ganze Zeit später bei einer Bewerbung, und dass man vielleicht überlegen sollte, inwieweit ein allzu schnelles Ermuntern der Lernenden, sich in der Zielsprache gleich der ganzen Welt mitzuteilen, tatsächlich so notwendig ist.

Trotzdem muss man festhalten, dass mit derartigen weltweit zugänglichen Publikationen die Möglichkeit gegeben ist, über die Grenzen des Klassenzimmers hinauszutreten, und je stärker es z.B. passiert, dass eine Gruppe von Lernenden ein digitales Produkt erstellt und dann in sozialen Netzwerken >werbend dafür sorgt, dass Leute, die keinen didaktischen Grund dafür haben ${ }^{1}$, dieses Produkt wahrzunehmen, es freiwillig zur Kenntnis nehmen, desto stärker finden Schritte über das Klassenzimmer hinaus in Richtung >natürliche Kommunikation< statt, die über die Interaktionsmöglichkeiten klassischer Projektdidaktik hinausgehen.

\subsection{Freie Lernertexte in einer didak- tischen Lernumgebung}

Während der Text in Abb. 1 ein Beispiel dafür ist, wie Lernende die digitalen Medien dafür nutzen, ohne didaktischen Kontext sich in der Fremdsprache anderen mitzuteilen (und $\mathrm{zu}$ hoffen, dass irgendwo in der Welt >draußen diesen Text als für sich so relevant empfindet, dass er auf ihn antwortet), ist Abb. 2 ein Beispiel für weitgehend freies Schreiben in einem didaktischen Raum.

Abb. 2 zeigt die Startseite der interaktiven Schreibwerkstatt der Lernumgebung für Jugendliche auf Mittelstufenniveau, Jetzt Deutsch Lernen. Diese Schreibwerkstatt stellt ein Wiki bereit, gibt Startinformationen und einen inhaltlichen Impuls. Danach sind die Lernenden freie Autoren, die mit anderen Autoren kooperieren. Verglichen mit dem völlig freien Text in Abb. 1 findet also eine Steuerung statt. In diesem konkreten Fall ist sie sehr gering, Ort, allgemeine Hilfestellung und Impuls werden gegeben (vgl. dazu ausführlicher Platten 2008).

Man kann sich derartige Wikis auch mit stärkeren Eingriffen vorstellen, z. B. mit einem Tutor, der textsortenbezogene Ratschläge gibt, kooperative Aktivitäten moderiert usw. Und man kann sich ein derartiges Wiki noch freier vorstellen, indem selbst der thematische Impuls

1 Also weder Lehrer, Partnerklasse, Eltern oder sonstige kommunikativ >zwangsverpflichtete< Personen sind. 


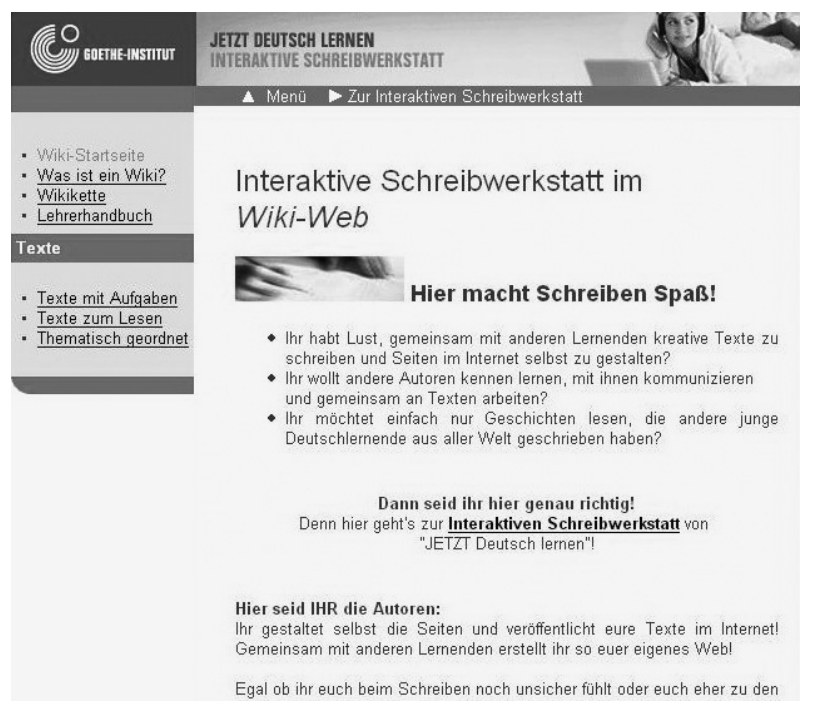

Abb. 2: Kooperatives Schreiben in einem didaktischen Kontext. http://www.goethe.de/z/jetzt/ dejwiki/dejwiki.htm (Abrufdatum 21.5.2008) noch weggelassen wird und lediglich ein bereitgestellter Schreibraum übrig bleibt. Unterschiedlich sein können auch die Motive der Schreiber: sind sie als individuelle Lerner in die Schreibwerkstatt geraten, sind sie von ihren Lehrern dazu >überredet w worden, ist gar eine ganze Lernergruppe als Teil des Unterrichts zu diesem >freien Schreiben verpflichtet worden? Hier bedarf es noch einer ganzen Reihe von Untersuchungen, bis man mehr darüber weiß, welcher Grad von Steuerung, thematischen Anreizen und evtl. auch unterrichtlichen Begleitungen für welche Lerner mit welchen Lernerfahrungen mit welchem Sprachstand für kooperatives Schreiben im Wiki angemessen ist.

\subsection{Integration von Lernertexten in Lehrmaterial}

Aufgrund der Bedeutung, die Lehrmaterial für das gesteuerte Fremdsprachenlernen hat, zumindest ebenso wichtig ist m. E. die Frage, ob es über diese erwei- terten Möglichkeiten des kooperativen freien Schreibens von Texten durch Lernende hinaus auch dazu kommen kann, im Lehrmaterial den Texten von Lernenden stärkeres Gewicht zukommen zu lassen. Lernertexte in Lehrwerken waren traditionell vereinzelte Texte von Lernenden, die z. B. auf ein Preisausschreiben geantwortet hatten. Mit der Flexibilität der digitalen Medien ist es nun möglich, diese Art der Integration von Lernertexten voranzutreiben. Abb. 3 zeigt ein weiteres Beispiel aus der Lernumgebung Jetzt Deutsch Lernen. Dort hatte man angefangen, Texte von Lernenden als Reaktionen auf die Texte der Lernumgebung ins Netz zu stellen, und ging danach einen Schritt weiter: die Texte der Lernenden wurden selbst zu didaktisch bearbeiteten Texten, die die Basis von Lerneinheiten bilden.

Man kann entweder sagen, damit seien sie genau so gut oder genauso schlecht wie alle anderen Lehrwerktexte, man kann aber auch hoffen, dass, wenn derar- 


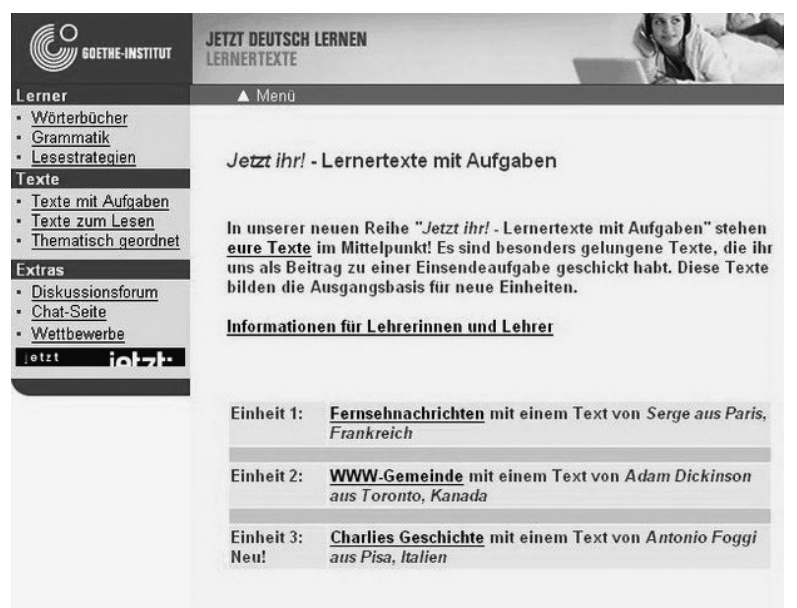

Abb 3: Didaktisierte Lernertexte im Lehrmaterial. http:// www.goethe.de/z/jetzt/dejlerte.htm (Abrufdatum 21.5.2008) tige Texte kontinuierlich erneuert, weitergesponnen werden usw., auch in Lehrwerken - wenn man sich, wie in Kap. 1 dargestellt, Lehrwerke als Datenbanken und nicht als statische Sequenzen vorstellt - mit diesen Texten eine stärkere Annäherung an Interessen der Lernenden erfolgen kann. Denn von den Lernenden kann ein für sie unangemessener Text in sihrem< Lehrwerk als Aufforderung verstanden werden, selbst aktiv zu werden, zu schreiben, zu inszenieren und diese Texte dann wieder anderen Lernenden zur Verfügung zu stellen, so dass in einem Datenbank-Konzept von Lehrwerk Texte von Lernenden gleichberechtigt neben von Materialmachern geschriebenen oder gefundenen stehen könnten, für andere und von anderen Lehrenden und Lernenden ausgewählt nicht danach, ob sie von berühmten Autoren, Lehrwerkmachern oder Lernenden stammen, sondern weil sie für bestimmte Lernende mit bestimmten Lernzielen zu einem bestimmten Zeitpunkt die angemessenen Texte sind. Und damit hätte man sich dann ein Stückchen weiter an so etwas wie >didaktische Au- thentizität< angenähert, an einen Authentizitätsbegriff, der nicht den Texturheber oder die Textbeschaffenheit verabsolutiert, sondern sich auf die komplexe Situation gesteuerten Fremdsprachenlernens einlässt.

\section{Literatur}

Barkowski, Hans; Fritsche, Michael; Göbel, Richard; von der Handt, Gerhard; Harnisch, Ulrike; Krumm, Hans-Jürgen; Kumm, Sigrid; Menk, Antje-Katrin; Nikitopoulos, Pantelis; Werkmeister, Manfred: Deutsch für ausländische Arbeiter. Gutachten zu ausgewählten Lehrwerken. 3., aktualisierte Auflage. Mainz: Werkmeister, 1986.

Bochow, Peter; Schroedter-Albers, Henning: »Deutsch Aktiv - Adaption für Indien; Didaktische Überlegungen, Unterrichtsbeispiele«, German Studies in India 1981, 173-188.

Engel, Ulrich; Krumm, Hans-Jürgen; Stickel, Gerhard; Wierlacher, Alois: Mannheimer Gutachten zu ausgewählten Lehrwerken Deutsch als Fremdsprache. Erstellt im Auftrag des Auswärtigen Amtes der Bundesrepublik Deutschland von der Kommission für Lehrwerke Deutsch als Fremdsprache, Band 2. Heidelberg: Groos, 1979. 
Fandrych, Christian; Tschirner, Erwin: »Korpuslinguistik und Deutsch als Fremdsprache. Ein Perspektivenwechsel«, Deutsch als Fremdsprache 44, 4 (2007), 195-204.

Funk, Hermann; Koenig, Michael: Grammatik lehren und lernen (Fernstudieneinheit 1). Berlin; München; Wien: Langenscheidt, 1991.

Gamper, Johann; Knapp, Judith: »A review of intelligent CALL-systems", Computer Assisted Language Learning 15, 4 (2002), 329-342.

Gerighausen, Josef; Seel, Peter: »Der fremde Lerner und die fremde Sprache. Überlegungen zur Entwicklung regionalspezifischer Lehr- und Lernmaterialien für Länder der Dritten Welt", Jahrbuch Deutsch als Fremdsprache 10 (1984), 126162.

Kommission für Lehrwerke DaF: Mannheimer Gutachten zu ausgewählten Lehrwerken Deutsch als Fremdsprache. Heidelberg: Groos, 1978.

Lemnitzer, Lothar; Naumann, Karin: »)Auf Wiederlesen! - - das schriftlich verfaßte Unterrichtsgespräch in der computervermittelten Kommunikation«. In: Beißwenger, Michael (Hrsg.): Chat-Kommunikation - Sprache, Interaktion, Sozialität \& Identität in synchroner computervermittelter Kommunikation. Perspektiven auf ein interdisziplinäres Forschungsfeld. Stuttgart: ibidem, 2001, 469-491.

Ludewig, Petra: Korpusbasiertes Kollokationslernen: Computer-Assisted Language Learning als prototypisches Anwendungsszenario der Computerlinguistik. Berlin; Bern; Brüssel; Frankfurt a. M.; New York; Oxford; Wien: Lang, 2005.

Lüdeling, Anke; Doolittle, Seanna; Hirschmann, Hagen; Schmidt, Karin; Walter, Maik: »Das Lernerkorpus Falko «, Deutsch als Fremdsprache 45, 2 (2008), 67-73.

Meißner, Cordula: »Eine gebrauchsorientierte Beschreibung des Sprachsystems mit Hilfe der Korpuslinguistik - das Beispiel der Synonyme ewig und unendlich «, Deutsch als Fremdsprache 45, 1 (2008), 8-13.

Müller-Hartmann, Andreas: »Wenn sich die Lehrenden nicht verstehen, wie sollen sich dann die Lernenden verstehen? Fragen nach der Rolle der Lehrenden in global vernetzten Klassenräumen«. In: Bredella, Lothar; Christ, Herbert; Legutke, Michael K. (Hrsg.): Fremdverstehen zwischen Theorie und Praxis. Arbeiten aus dem Graduierten-Kolleg »Didaktik des Fremdverstehens«. Tübingen: Narr, 2000, 275-301.

Mukherjee, Joybrato: »Bridging the Gap between Applied Corpus Linguistics and the Reality of English Language Teaching in Germany«. In: Connor, Ulla; Upton, Thomas (Hrsg.): Applied Corpus Linguistics: A Multidimensional Perspective. Amsterdam: John Benjamins, 2004, 239-250.

Mukherjee, Joybrato; Rohrbach, Jens-Martin: »Rethinking Applied Corpus Linguistics from a Language-pedagogical Perspective: New Departures in Learner Corpus Research«. In: Kettemann, Bernhard; Marko, G. (Hrsg.): Planning, Gluing and Painting corpora: Inside the Applied Corpus Linguist's Workshop. Berlin; Bern; Brüssel; Frankfurt a. M.; New York; Oxford; Wien: Lang, 2006.

Nagata, Noriko: »Intelligent computer feedback for second language instruction«, The Modern Language Journal 77, 3 (1993), 330-339.

Neuner, Gerhard: »Lehrwerkanalyse und -kritik als Aufgabenfeld der Fremdsprachendidaktik - zur Entwicklung seit 1945 und zum gegenwärtigen Stand «. In: Neuner, Gerhard (Hrsg.): Zur Analyse fremdsprachlicher Lehrwerke. Berlin; Bern; Brüssel; Frankfurt a. M.; New York; Oxford; Wien: Lang, 1979, 5-39.

O'Dowd, Robert; Ritter, Markus: »Understanding and Working with Failed Communication in Telecollaborative Exchanges«, CALICO Journal 23, 3 (2006), 120.

Platten, Eva: „Chat-Tutoren im didaktischen Chat-Raum - Sprachliche Hilfen und Moderation«. In: Legutke, Michael K.; Rösler, Dietmar (Hrsg.): Fremdsprachenlernen mit digitalen Medien. Tübingen: Narr, 2003, 145-177.

Platten, Eva: »Gemeinsames Schreiben im Wiki-Web. Aktivitäten in einer untutorierten Schreibwerkstatt für fortgeschrittene Deutschlernende«, Zeitschrift für Interkulturellen Fremdsprachenunterricht 13, 1 (2008), 22 S. 
Pujolà, Joan-Tomàs: »Did CALL feedback feed back? Researching learners' use of feedback «, ReCALL 13, 1 (2001), 79-98.

Puskas, Csilla: Interaktives E-Learning im Bereich Deutsch als Fremdsprache: Fehleranalyse und intelligente Rückmeldung durch Parsing-Systeme. Gießen, 2008: http:// www.uni-giessen.de/ gm1160/doku/ cpu-master-thesis.pdf (Abrufdatum 21.05.2008)

Roche, Jörg; Scheller, Julija: »Zur Effizienz von Grammatikanimationen beim Spracherwerb: Ein empirischer Beitrag zu einer kognitiven Theorie des multimedialen Fremdsprachenerwerbs«, Zeitschrift für Interkulturellen Fremdsprachenunterricht 9, 1 (2004), 15pp.

Rösler, Dietmar: Lernerbezug und Lehrmaterial Deutsch als Fremdsprache. Voraussetzungen für die Adaption von konventionellem Material. Heidelberg: Groos, 1984.

Rösler, Dietmar: "Autonomes Lernen? Neue Medien und altes Fremdsprachenlernen «, Info DaF 25, 1 (1998), 3-20.

Rösler, Dietmar: E-Learning Fremdsprachen. Eine kritische Einführung (Stauffenburg Einführungen, 18). 2. Auflage, Tübingen: Stauffenburg, 2007.

Rösler, Dietmar: »Zwischen Lehrwerk und Online-Sprachbad. Zur Neubestimmung von Funktion und Gestalt von Lehrmaterialien im Anfängerunterricht«. In: Krumm, Hans-Jürgen; Portmann-Tselikas, Paul R. (Hrsg.): Theorie und Praxis. Österreichische Beiträge zu Deutsch als Fremdsprache. Innsbruck: Studienverlag, 2006a, 143-154.

Rösler, Dietmar: »Das scheinbare Paradox des Fremdsprachenlernens mit digitalen Medien: Tendenz zur Individualisierung und zur Stärkung kooperativer Lernformen«. In: Scherfer, Peter; Wolff, Dieter (Hrsg.): Vom Lehren und Lernen fremder Sprachen: eine vorläufige Bestandsaufnahme. Berlin; Bern; Brüssel; Frankfurt a. M.; New York; Oxford; Wien: Lang, 2006b, 157-169.

Rösler, Dietmar: »Lernziel kommunikative Kompetenz dreiunddreißig Jahre nach Piepho 1974 - ein kritischer Rückblick aus der Perspektive des Deutschlernens außerhalb des deutschsprachigen Raums«. In: Legutke, Michael K. (Hrsg.): Kommunikative Kompetenz als fremdspra- chendidaktische Vision. Tübingen: Narr, 2008, 115-129.

Schmidt, Torben: Gemeinsames Lernen mit Selbstlernsoftware im Englischunterricht Eine empirische Analyse lernprogrammgestützter Partnerarbeitsphasen (Giessener Beiträge zur Fremdsprachenforschung). Tübingen: Narr, 2007 (zugleich Dissertation an der Johann Wolfgang GoetheUniversität Frankfurt a. M. 2007).

Tamme, Claudia: E-Mail-Tutorien: eine empirische Untersuchung E-Mail-vermittelter Kommunikationen von Deutschstudierenden und Deutsch-als-Fremdsprache-Lehrenden in der Ausbildung (2001). Online: http:// geb.uni-giessen.de/geb/volltexte/2003/ 1009/ (Abrufdatum 21.05.2008).

Würffel, Nicola: »Und wenn die Wellenlänge nicht stimmt? Zum Einfluss affektiver Faktoren auf Verstehensprozesse in elektronischen Lehr-Lernsituationen (Elektronisches Praktikum)«, Fremdsprachen und Hochschule 72 (2004), 7-25.

Würffel, Nicola: Strategiegebrauch bei Aufgabenbearbeitungen in internetgestütztem Selbstlernmaterial (Giessener Beiträge zur Fremdsprachendidaktik). Tübingen: Narr, 2006.

Würffel, Nicola: »Kooperatives Schreiben im Fremdsprachenunterricht: Potentiale des Einsatzes von Social-Software-Anwendungen am Beispiel kooperativer OnlineEditoren«, Zeitschrift für Interkulturellen Fremdsprachenunterricht 13, 1 (2008), 26 S.

\section{Dietmar Rösler}

Von 1986 bis 1996 Hochschullehrer am Department of German des King's College der University of London. Seit 1996 Professur für Deutsch als Zweit- und Fremdsprache an der Justus-Liebig-Universität Gießen. Forschungsschwerpunkte: das Verhältnis von gesteuertem und natürlichem Zweit- und Fremdsprachenlernen, Lehrmaterialanalyse, Interkulturelle Kommunikation, Grammatikvermittlung und Sprachtechnologie und Fremdsprachenlernen. Ausführliche Informationen finden sich unter: http:// www.uni-giessen.de / cms / fbz / fb05/ germanistik/iprof/daf/ 